\title{
El estatus jurídico de la ciudad de Jerusalén y sus implicancias en el derecho internacional
}

\author{
The legal status of the city of Jerusalem and its implications in international
}

Martín CANEPA

Abogado. Jefe de trabajos prácticos en Derecho de la Integración. Facultad de Derecho, Universidad de Buenos Aires.

Fecha de recepción: 12 febrero de 2019 Fecha de aprobación: 30 de junio de 2019

Para citar este artículo / To reference this article Canepa, M. (2018) El estatus jurídico de la ciudad de jerusalén y sus implicaciones en el derecho internacional. Inciso, 21(1) ; 4-18.

DOI: http://dx.doi.org/10.18634/incj.21v.11.899

\section{Resumen}

El presente trabajo se propone analizar el conflicto actual en torno a la ciudad de Jerusalén a través de los diferentes elementos que constituyen el centro principal de la controversia, entre ellos, los elementos jurídico, religioso, político e histórico. El conflicto, a su vez, enfrenta a dos sujetos distintos, por un lado un Estado, el Estado israelí y por otro a la Autoridad Palestina, que todavía no es reconocida como un Estado con todos los derechos y obligaciones que tal condición implica (Mouton, 1996, p.967). La metodología utilizada consistirá en el análisis de fuentes históricas, datos actuales de organizaciones internacionales y doctrina de la Corte Internacional de Justicia. La conclusión más significativa, extraída de los resultados del presente trabajo es la confirmación de que el conflicto en torno a la ciudad debería solucionarse teniendo en cuenta, las posiciones de los dos actores involucrados, las necesidades de la población y los antecedentes de las negociaciones llevadas a cabo hasta el momento.

Palabras clave: - Palestina - Israel - Estatus jurídico -Ocupación. 


\begin{abstract}
The present work intends to analyze the current conflict around the city of Jerusalem through the different elements that constitute the main center of the controversy, including the legal, religious, political and historical elements. The conflict, in turn, confronts two different subjects, on the one hand a State, the Israeli State; on the other, the Palestinian Authority, which is not yet recognized as a State, with all the rights and obligations that such a condition implies (Mouton, 1996, p.967). The methodology used here (consists) of the analysis of historical sources, current data of international organizations, and doctrine of the International Court of Justice. The most significant conclusion, drawn from the results of the present work, is the confirmation that the conflict around the city should be resolved taking into account the positions of the two parts involved, the needs of the population, and the background of the negotiations so far carried out.
\end{abstract}

Keywords: Palestine - Israel - Legal status - Occupation.

\title{
INTRODUCCIÓN
}

Se intentará explicar los motivos del conflicto, revisando acontecimientos históricos, describiendo el estatus actual de la ciudad y resaltando los riesgos de división; se realizará un resumen de las negociaciones internacionales más relevantes al respecto, y la presentación de un breve esquema de los puntos de acuerdo y desacuerdo en relación con las posibles soluciones que se han vislumbrado hasta el momento. Luego se destacará las implicancias que produce esta situación conflictiva en relación con la comunidad internacional y al Derecho Internacional Público. Finalmente se hará referencia a la construcción del Muro en territorio palestino ocupado.

\section{Motivos DEL CONFLICTO}

La ciudad de Jerusalén no es una ciudad más, sino que representa el centro de convergencia de las tres religiones monoteístas más influyentes del mundo. La ciudad vieja es precisamente el punto más conflictivo y que genera la mayor parte de disputas. La importancia que se le asigna desde el punto de vista religioso se basa en el hecho de que los acontecimientos más importantes que narran los principales textos religiosos sitúan en ella una serie de elementos que la colocan en un marco de santidad incomparable en relación con otras ciudades.

En cuanto al cristianismo, allí se encuentra el Santo Sepulcro, el lugar, hoy constituido en iglesia a partir de la decisión del emperador Constantino, en donde se cree que fue sepultado Jesús y donde posteriormente resucitó. Para el judaísmo la importancia reside entre otros fundamentos, en el Muro de los Lamentos o Muro Occidental. Es el último vestigio del antiguo Templo de Jerusalén, en donde se considera que se guardaba el Arca 
de la Santa Alianza, un cofre que contenía las tablas de la ley con los diez mandamientos. Para el Islam, Jerusalén es una de las ciudades sagradas más importantes junto con la Meca y Medina ya que, según el Corán, Mahoma ascendió al trono de Alá desde Jerusalén luego de un viaje nocturno iniciado en Medina.

Las partes involucradas en el conflicto son el Estado de Israel y la Autoridad Palestina. El valor religioso que se le asigna a la ciudad ha tenido una influencia crucial en los reclamos de las partes mencionadas, en especial por parte de Palestina. Esta considera que la futura capital de su Estado debe estar situada en Jerusalén y en caso de que no sea en la totalidad de la ciudad, la capital debería situarse en la parte Este. Israel por su parte no ve ninguna disputa en este sentido, ya que ejerce actos de soberanía sobre toda la ciudad y una división de la misma implicaría romper el equilibrio y la integridad del Estado judío.

Para poder comprender más acertadamente el conflicto es importante hacer referencia a ciertos acontecimientos históricos que marcaron sus rasgos más característicos. Jerusalén es una ciudad que se encuentra atravesada por la confrontación étnica nacional de dos grupos: árabes y judíos. En comparación con otras ciudades multiculturales que atraviesan el mismo problema como Bruselas o Montreal, en Jerusalén, no existen instituciones que se encarguen de apaciguar la confrontación y de esta manera disminuir los grados de tensión entre ambos grupos. Para ciertos autores (Klein, 2001), la administración de la ciudad se ha concentrado más en gobernar y ejercer efectivamente el poder en toda la ciudad, pero no en llevar a cabo tareas de conciliación. Es la ciudad israelí más densamente poblada. Luego de la guerra de los seis días, Israel anexó Jerusalén Este, pero incluye no solo la parte jordana de la ciudad que cubría 6.5 km, sino también 64.4 km adicionales de Cisjordania y parte de Belén y Beit Jala.

El Estado israelí se ha orientado en dos líneas para lograr la ocupación efectiva sobre Jerusalén Este: aumentar la población judía y llevar la construcción a gran escala de viviendas en las áreas árabes para de esta manera crear un cambio demográfico irreversible. A su vez, en 1973 la Administración de tierras de Israel creó una unidad llamada Igum (Klein, 2001) encargada de comprar propiedades que luego se convertirían en instituciones públicas judías en la ciudad vieja. La confiscación a gran escala de tierras de árabes también contribuyó a este plan. Hoy en día solo el 7,3 \% de Jerusalén Este está habilitada para la construcción por parte de palestinos a causa de restricciones legales impuestas por parte del gobierno local, lo que contribuye a lograr beneficios en favor de la población judía.

Los Acuerdos de Oslo II $^{1}$, firmados en septiembre de 1995, dividieron a Jerusalén en cuatro zonas de control. La zona israelí incluía la ciudad de Jerusalén y los asentamientos israelíes que la rodeaban. La palestina se encontraba a su vez dividida en la A y en la B. 
La A estaba completamente bajo la Autoridad Palestina e incluía las ciudades de Jericó, Belén, Beit Jala, Ramalá y Al-Bireh, las cuales están situadas en la periferia de Jerusalén. En la Zona B a diferencia de la anterior, los palestinos tenían un control civil y de policía, pero la responsabilidad por la seguridad de este sector estaba a cargo de Israel. La B incluía ciertos suburbios de Jerusalén como Al -Azaria, Abu Dis, y Hizma. Los palestinos y Gran Bretaña rechazaron desde un principio la partición de la ciudad.

La Comisión de las Naciones Unidas para la cuestión de Palestina, Unscop, fue establecida en mayo 1947 y a fines de agosto envió sus recomendaciones a la Asamblea General de las Naciones Unidas (Virally, 1963). La Comisión no aceptó la posición del movimiento sionista que otorgaba soberanía israelí sobre Jerusalén occidental y sometía a la parte Este de la ciudad a un régimen de administración internacional. La recomendación consistía en dividir la antigua Palestina en dos Estados, uno judío y otro palestino y colocar a la totalidad de la ciudad de Jerusalén bajo un régimen internacional. A través de la resolución $181^{2}$ de la Asamblea General de las Naciones Unidas, se recomendó la aplicación de un plan que incluía la internacionalización de Jerusalén y la división de Palestina en dos estados. Esta solución implicaba la desmilitarización y neutralidad de la ciudad. Los países árabes rechazaron inmediatamente la propuesta de partición y en consecuencia los representantes del futuro Estado judío consideraron que no estaban obligados a cumplir con la resolución que implicaba la división de Palestina y la internacionalización de la ciudad santa. El 5 de diciembre de 1948, Ben-Gurion reclamó a Jerusalén como parte de Israel y ocho años más tarde la Knesset declaró a Jerusalén como su capital eterna e indivisible. Jerusalén Este fue anexada al Reino de Jordania en 13 diciembre de 1948. Finalmente, en 1952 la Asamblea General de las Naciones Unidas decidió que Israel y Jordania serían responsables de llegar a un acuerdo sobre la ciudad de Jerusalén.

\section{La DIVISIÓN DE LA CIUDAD}

Uno de los reclamos de Palestina consiste en que se considere a Cisjordania y Jerusalén Este como una sola unidad, de manera tal que se logre una idéntica solución política y diplomática para esta zona (Shragai, 2010). Para muchos israelíes la partición de la ciudad traería varios riesgos (Shragai, 2012). Por un lado, el cambio de estatus de algunos barrios provocaría que cerca de diez mil judíos tengan que dejar la ciudad como sucedió luego de 1948, cuando un cuarto de la población judía de Jerusalén, alrededor de 25.000 personas fueron desplazadas de la ciudad.

Uno de los puntos más controvertidos y que ha atraído la atención de gran parte de la comunidad internacional en el último tiempo, es la zona denominada E-1 (East 1). Esta zona, se encuentra incluída, de acuerdo con Israel, dentro del radio de la ciudad israelí de Maale Adumim, que cuenta con 36.000 habitantes. Es una zona inmediatamente

2. Asamblea General de las Naciones Unidas. Resolución 181 (II), 29 de noviembre de 1947 sobre futuro Gobierno de Palestina. 
adyacente a Jerusalén Este que está mayormente deshabitada y en gran parte se trata de tierras que son propiedad del Estado. El Ministerio israelí de vivienda ha autorizado la construcción en este sector para poder unir la ciudad de Maale Adumim con Jerusalén. Esta autorización provocó la queja de Palestina, ya que considera que la construcción en esta zona traerá como consecuencia la separación de Cisjordania en dos y afectará la contigüidad del futuro Estado palestino. Por su parte Israel ha declarado que para que esto no suceda, planea la construcción de una ruta que permitirá el tráfico de palestinos que vienen del sur a través del extremo Este de Maale Adumim, continuando hacia el norte de manera tal que pueda conectarse con las ciudades del Norte de Cisjordania. A su vez, Israel argumenta que la zona comprendida en E-1, se encuentra dentro de lo que se denomina Zona C, en virtud de las disposiciones del Acuerdo de Oslo II y sobre la cual Israel retuvo competencias de control y planificación. Asimismo, sostiene que ninguno de los acuerdos de Oslo prohíbe que Israel construya asentamientos allí.

\section{ACUERDOS Y DESACUERDOS}

Antes de introducir el análisis de los temas específicos sobre los que versa la disputa territorial y acerca de los cuales fue posible encontrar un acuerdo, es relevante enumerar una síntesis de las negociaciones llevadas a cabo durante el período comprendido entre 1993-2011:

- Acuerdos de Oslo ${ }^{3}$ firmados entre Israel y La Organización para la Liberación de Palestina (OLP): ambas partes se comprometen a negociar la cuestión de Jerusalén.

- 1999-2000: durante la administración de Ehud Barak se llevaron a cabo en forma efectiva varias negociaciones.

- Cumbre de Camp David4: se introduce la cuestión de Jerusalén en las negociaciones. Sin embargo, fracasa a causa de la disputa en torno a la soberanía sobre el Monte del templo.

- Gobierno de Ariel Sharon (2001 - 2006): no se realizaron negociación sobre Jerusalén.

- Cumbre de Annapolis 5 : a pedido del equipo negociador israelí, la cuestión de Jerusalén fue descartada, la cual solo se trató en los encuentros privados entre el primer ministro Olmert y el representante de la Autoridad Palestina Abu Mazen. Estas negociaciones se paralizaron a partir de la operación militar Plomo Fundido 6 en Gaza.

3. Acuerdos de Oslo, 13 de septiembre de 1993. Los acuerdos fueron firmados como resultado de la Conferencia de Madrid de 1991.

4. Cumbre de paz en Oriente Medio (Camp David II), Julio 2000.

5. Conferencia de Annapolis, 27 de noviembre de 2007.

6. Operación militar llevada a cabo por las Fuerzas de Defensa Israelíes, 27 de diciembre de 2008-18 de enero de 2009. 
- Gobierno de Benyamin Netanyahu (marzo 2009 - hasta la actualidad): continuación de negociaciones para llegar a un acuerdo permanente, pero sin resultados concretos.

A continuación, haremos una breve descripción de los puntos (Lehrs, 2011) en los cuales sí hubo algún acuerdo y en los cuales no, en los siguientes aspectos: barrios de Jerusalén Este, la Ciudad vieja, el Muro de los Lamentos y el Monte del Templo.

\section{a. Barrios de Jerusalén Este}

Durante la fórmula propuesta por Clinton y luego también en las reuniones llevadas a cabo en Bolling Air Force Base (2000) y Taba (2001), se aceptó la posibilidad de que cada parte tendría soberanía sobre los respectivos barrios, judío y árabe, respectivamente, ambos ubicados en Jerusalén Este. El principal obstáculo fue la negación por parte de Palestina a reconocer soberanía sobre el barrio de Har Homa, el cual fue construido luego de los Acuerdos de Oslo. A su vez, también constituyeron un impedimento, las cuestiones de transporte y geografía que podrían llegar a romper la continuidad con otros barrios.

\section{b. La ciudad vieja}

Aquí la cuestión tomó dos direcciones. Por un lado, la posibilidad de dividir la soberanía o la de establecer un régimen internacional. En principio, la parte Israelí prefería una solución basada en un "régimen especial" que no implicara una división. Mientras que los palestinos por su parte sí exigían un estatus compartido. Durante la administración de Ehud Brak se acordó que el barrio judío estaría bajo la administración israelí mientras que el barrio musulmán y el barrio cristiano estarían bajo la palestina. Los desacuerdos quedaban expuestos en el punto relativo al espacio comprendido por el barrio armenio y áreas fuera de los límites de la ciudad vieja como la Ciudad de David y el Monte de los Olivos. Israel exigía administrar estos territorios mientras que Palestina la reclamada también para sí, pero permitía que fueran administrados por Israel. El mismo desacuerdo se verificaba respecto del túnel del Muro de los Lamentos y la Torre de David.

Durante la Cumbre de Annapolis ambas partes parecen haber acordado la creación de una especie de Autoridad Internacional compuesta por Israel, Palestina, Estados Unidos, Jordania y Arabia Saudita, con el propósito de administrar ciertos sectores "sagrados" dentro de la ciudad vieja. Los desacuerdos surgieron a raíz de las aéreas específicas que estarían incluidas en este régimen.

\section{c. El Muro de los Lamentos y el Monte del Templo}

Durante la administración de Ehud Brak se acordó que el Muro de los Lamentos estaría bajo el control israelí y que el Monte del Templo quedaría bajo la esfera del futuro Estado 
Palestino. Los desacuerdos giraron en torno al Monte del Templo y a la demarcación del territorio sobre el cual Israel ejercería soberanía en relación con el Muro de los Lamentos.

\section{Efectos}

El conflicto entre Palestina e Israel por la ciudad de Jerusalén ha dividido desde sus orígenes a la comunidad internacional y le ha exigido que tome una posición al respecto. El Estatus actual (Berkovitz, 2001) de la ciudad implica tener en cuenta que Israel es el Estado que efectivamente ejerce soberanía y administra toda la ciudad incluyendo Jerusalén Este. Luego de la guerra de 1967, cuando Israel lleva a cabo la anexión de la parte Este, el Consejo de Seguridad ${ }^{7}$ de la ONU instó a la comunidad internacional para que no se reconociera esta situación. En consecuencia, la mayor parte de los Estados han retirado sus embajadas de Jerusalén y las han trasladado a Tel-Aviv. Sin embargo, existen algunos países, que aún hoy, conservan consulados en la ciudad de Jerusalén como por ejemplo los Estados Unidos, el cual ha ido un paso más adelante al trasladar su embajada en el año 2018. Esta situación plantea ciertas incongruencias en relación a la diplomacia internacional y a la decisión de no reconocer la efectiva ocupación de Israel sobre Jerusalén Este, ya que para instalar un consulado en un país extranjero se necesita la autorización del Estado en cuyo territorio se quiere instalar e implica para ciertos autores reconocer de facto la soberanía de ese Estado sobre dicho territorio. En la mayoría de los casos los Estados que poseen consulados dejan a salvo sus observaciones respecto del no reconocimiento de la situación de hecho producida luego de la guerra de 1967.

Es importante en este punto plasmar la opinión de algunos doctrinarios, por ejemplo, Dore Gold (Gold, 2007), quien sostiene que la anteriormente mencionada resolución 242 del Consejo de Seguridad plantea un juego de palabras que pone de relieve las discusiones diplomáticas que rodearon el texto de la misma. En primer lugar, la resolución fue adoptada en el marco del capítulo VI de la Carta de las Naciones Unidas, el cual trata del arreglo pacífico de controversias, iniciación de negociaciones, arbitraje y mediación. Estas resoluciones no son consideradas obligatorias por sí mismas. Por lo tanto, para que esta resolución se hubiera considerado obligatoria, se tendría que haber adoptado en el marco del capítulo VII de la Carta que se ocupa de los actos de agresión y amenaza a la paz internacional. Ya al analizar en el texto de la resolución, la misma establece en su inciso 1.(i) que es necesario establecer una paz final y duradera en Medio Oriente, la cual debe incluir: Retiro de las fuerzas armadas israelíes de territorios ocupados en el reciente conflicto.

Dore Gold argumenta que, a pesar de la posición de ciertos Estados, por ejemplo, la entonces Unión Soviética, quien a través de su Primer Ministro Alexei Kozygin, sostenía que el texto explícitamente fue redactado haciendo referencia a "todos" Ios territorios

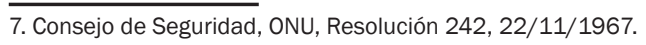


ocupados, se plasmó solo la palabra "territorios". A su vez, se excluyó el artículo definido "los" (the) del texto negociado íntegramente en inglés. Como consecuencia, la redacción final implicaría para Gold que Israel no deberá retirarse de todos los territorios ocupados. Adicionalmente, considera que si los Estados que adoptaron la resolución en el seno del Consejo de Seguridad hubieran querido incluir expresamente dentro de aquellos territorios respecto de los cuales se debía retirar Israel, a Jerusalén Este, lo hubieran hecho.

Como hemos podido observar en el texto de la resolución, la ciudad de Jerusalén no fue mencionada. Sin embargo, no hay que soslayar el hecho de que la Carta de las Naciones Unidas en su artículo 2.4 prohíbe expresamente el empleo de la fuerza contra la integridad territorial o la independencia política de todo Estado, lo cual implica el uso de la fuerza o de líneas de demarcación y otras delimitaciones provisorias, la ocupación y la anexión de territorios. De este principio, se deduce la consiguiente inadmisibilidad de la adquisición de espacios territoriales como consecuencia de actos de guerra. Por lo tanto, la ocupación militar prolongada en el tiempo en sectores palestinos constituye una violación grave a los principios de derecho internacional que consagran el no uso de la fuerza y el reconocimiento de la soberanía estatal (Kuete Minga, 1997).

La mayor parte de los Estados árabes, salvo Egipto y Jordania, con los que Israel ha firmado tratados de paz, no reconocen la existencia del Estado de Israel, por lo que la situación es aún más compleja en estos casos. Israel limita con el Líbano y Siria y ambos países no lo reconocen como Estado, lo que implica que no solo el comercio entre ellos esté paralizado, sino cualquier otro tipo de actividad como la circulación de personas y de servicios.

La situación actual del estatus de la ciudad, supone reconocer que la misma se encuentra bajo la soberanía de Israel, y en los hechos es la capital del Estado, ya que sus principales órganos de gobierno, Poder Ejecutivo, Poder Judicial (Corte Suprema) y Poder Legislativo (Knesset) están situados en la ciudad de Jerusalén. Israel administra y aplica su ley en el territorio de la ciudad incluyendo Jerusalén Este. Es importante reconocer que a pesar de estas circunstancias, la administración de los sitios religiosos para el Islam, en el Monte del Templo, no es ejercida por el gobierno israelí. Luego de la guerra de los seis días de 1967 Jordania comenzó a tener un rol de Autoridad Administrativa en estos lugares sagrados, representando de esta manera el interés del mundo musulmán (Gold, 2007). Esta cooperación se consolidó en 1994 con el acuerdo entre Israel y Jordania, conocido como la Declaración de Washington ${ }^{8}$ que fue incorporada al Tratado de Paz firmado entre ambos Estados. La misma establece en su inciso B.3:

8. Washington Declaration between Israel-Jordan-United Estates, 25 th July 1994. 
Israel respeta el actual rol especial del Reino Hachemita de Jordania en los sitios sagrados musulmanes en Jerusalén. Cuando las negociaciones acerca del estatus permanente tengan lugar, Israel dará prioridad al histórico rol de Jordania sobre aquellos sitios. Asimismo, ambas partes acuerdan actuar conjuntamente para promover relaciones entre las tres religiones monoteístas ${ }^{9}$

De acuerdo con la Herzliya Conference de 2006 (Kimhi, Choshen, Assap-Shapira, 2006, p.9) hay ciertos cambios que ha sufrido Jerusalén y que tienen que ser tenidos en cuenta:

A raíz de la baja en la natalidad de familias judías, se cree que en 25 años aproximadamente, la ciudad habrá perdido la mayoría de los habitantes judíos que la caracterizó desde la segunda mitad del siglo XIX. Esta situación tendría efectos desde el punto de vista geopolítico para Israel.

El balance negativo de la inmigración hacia la ciudad, que se experimenta desde los inicios de 1980, daña la imagen de la misma y no contribuye positivamente a mejorar su estatus social.

La economía se encuentra en un período de decadencia. De acuerdo a índices socio-económicos de la Oficia Central de Estadísticas del Banco Central de Israel, la situación económica de Jerusalén se ha visto deteriorada en la última década. En este sentido, la ciudad es considerada como la más pobre en comparación con otras grandes ciudades de Israel. (Kimhi, Choshen, Assap-Shapira, 2006, p.9)

Estos problemas actuales que atraviesa hacen repensar a las autoridades israelíes sus planes de gobierno y las acciones a tomar para evitar que se produzcan consecuencias indeseables, por ejemplo, la baja de población judía, que traería como resultado una mayoría de población árabe. Esto a su vez provocaría un cambio en la composición del conjunto de electores al momento de las elecciones, por lo tanto, una modificación de los representantes locales que luego se trasladaría a nivel nacional.

\section{La construcción del muro y la Corte Internacional de Justicia}

Desde el punto de vista jurídico el conflicto ha llamado la atención de las Naciones Unidas, la cual a través de la Asamblea General ha intentado vislumbrar una solución legal al problema. En consecuencia, la Asamblea solicitó una Opinión consultiva ${ }^{10}$ para que la Corte se pronuncie acerca de las consecuencias jurídicas de la construcción de un muro en el territorio palestino ocupado. A través de la resolución ES-10/14, adoptada el 8 de diciembre de 2003, instó al Tribunal para que dé respuesta a la siguiente pregunta ${ }^{11}$ :

9. Traducción libre del autor. Texto original en inglés: "Israel respects the present special role of the Hashemite Kingdom of Jordan in Muslim Holy shrines in Jerusalem. When negotiations on the permanent status will take place, Israel will give high priority to the Jordanian historic role in these shrines. In addition the two sides have agreed to act together to promote interfaith relations among the three monotheistic religions".

10. ICJ, Legal Consequences of the Construction of a Wall in the Occupied Palestinian Territory, Advisory Opinion, 2004.

11. Traducción libre del autor. Texto original en francés: “Quelles sont en droit les conséquences de l'édification du mur qu'lsraël, puissance occupante, est en train de construire dans le territoire palestinien occupé, y compris à l'intérieur et sur le pourtour de Jérusalem-Est, selon ce qui est exposé dans le rapport du Secrétaire général, compte tenu des règles et des principes du droit international, notamment la quatrième convention de Genève de 1949 et les résolutions consacrées à la question par le Conseil de sécurité et l'Assemblée générale ?" 
¿Cuáles son las consecuencias jurídicas de la edificación del muro que Israel, potencia ocupante, está construyendo en el territorio palestino ocupado, incluyendo en su interior y contorno a Jerusalén Este, de acuerdo a lo expuesto en el reporte del Secretario General, teniendo en cuenta las reglas y principios del derecho internacional, en especial la cuarta Convención de Ginebra de 1949 y las resoluciones consagradas a este caso por el Consejo de Seguridad y la Asamblea General?

Cuando la Corte resuelve con base a su competencia consultiva (Esposito, 1996), su decisión no es obligatoria como en el caso de una sentencia, sino que tienen el efecto de una recomendación. Sin embargo, las opiniones consultivas, al emanar del más alto tribunal en materia de Derecho Internacional, tienen un valor doctrinario que marca el rumbo de muchas de sus decisiones posteriores e incluso de otros tribunales internacionales.

En el caso en cuestión, la Corte debía limitarse a responder la pregunta para la cual fue llamada a decidir, no se trataba de un caso contencioso. Sin embargo, muchos de los párrafos del texto de la opinión, parecieran condenar al Estado de Israel por ciertos actos que se le atribuyen. En este sentido, la Corte consideró que Israel, como potencia ocupante, tiene ciertos deberes y obligaciones y ha violado normas del Derecho Internacional Humanitario y del Derecho Internacional de los Derechos Humanos a raíz de la construcción del muro en contra de estos deberes y obligaciones.

Respecto de las normas de Derecho Internacional Humanitario, según la Corte, ha llevado a cabo los siguientes actos contrarios a las mismas:

Prohibición de modificar la composición demográfica del territorio palestino ocupado.

Destrucción de propiedad privada en contra de las disposiciones de los artículos 46 y 52 del Reglamento de la Haya de 1907 y el artículo 53 de la cuarta Convención de Ginebra.

A su vez, también han llevado a cabo actos contrarios al Derecho Internacional de los Derechos Humanos, a saber:

Restricción de la libertad de elección del lugar de residencia respecto de los palestinos.

La construcción del Muro provocó graves limitaciones del derecho a trabajar, a la salud y a la educación, dificultades para el acceso a establecimientos escolares y aprovisionamiento de agua.

Antes de finalizar con el análisis de las cuestiones resueltas por la Corte en la mencionada.

Opinión consultiva, no se deben soslayar algunas de las opiniones individuales de los jueces intervinientes y la única disidencia. En este sentido, el juez Koroma suscribe la decisión de la Corte, pero pone de relieve determinados puntos de la misma. Afirma que la edificación del muro conlleva la violación del principio fundamental de Derecho 
Internacional, que prohíbe la adquisición de territorios por la fuerza. Como consecuencia de este principio, Israel no está habilitado a llevar a cabo actos de soberanía que tengan por efecto modificar el estatus del territorio ocupado (Poissonnier y Osseland, 2010). Resalta la conclusión de la Corte en relación al derecho de autodeterminación, reconocido por el Derecho Internacional, como aplicable tanto al territorio como al pueblo palestino. La construcción del muro sobre territorio palestino obstaculizaría la concreción de este derecho. Finalmente, considera que la comunidad internacional tiene la obligación de no reconocer ninguna modificación unilateral del estatus del territorio ocupado, que podría tener lugar como corolario de la construcción del muro.

Por su parte, la jueza Higgings deja en claro que, a pesar del estatus especial de Palestina, que no puede considerarse todavía un Estado independiente, le es permitido participar del procedimiento ante la Corte, existiendo un diferendo entre dos actores internacionales. En relación al derecho a la autodeterminación, no concuerda con la Corte, en el sentido de que la construcción del muro constituiría un obstáculo grave al ejercicio de este derecho, sino que el verdadero obstáculo sería la incapacidad manifiesta y la falta de voluntad tanto de Israel como de Palestina de actuar para permitir que el pueblo palestino logre la autodeterminación. Se pregunta por qué resulta contrario al Derecho Internacional, que una potencia ocupante pierda el derecho a defender a sus propios ciudadanos frente a ataques que provengan del territorio ocupado. Consecuentemente, resulta incoherente, que Palestina tenga capacidad internacional para ser invitada al procedimiento consultivo, beneficiarse de las disposiciones que emanan del derecho internacional humanitario, pero no ser una entidad internacional con obligaciones que le impongan una prohibición de llevar a cabo ataques armados contra otros sujetos de Derecho Internacional.

Finalmente, el único voto en disidencia es el del juez Thomas Buergenthal, quien considera que la Corte no ha examinado la naturaleza de los atentados llevados a cabo desde el otro lado de la línea verde sobre Israel y su población. En cuanto a la posibilidad de que el Estado israelí tenga el derecho a la legítima defensa, es necesario examinar la naturaleza de los atentados provocados por terroristas contra Israel y si la construcción del muro en todo o en parte podría constituir una respuesta necesaria y proporcionada a este tipo de agresiones. La Corte tendría que haberse negado a conocer del asunto, ya que faltan elementos de hecho concernientes a la construcción del muro. Israel no ha presentado la suficiente información y la Corte se ha basado exclusivamente en los reportes de las Naciones Unidas. Estos elementos faltantes, permitirían demostrar las razones para justificar los imperativos militares o las necesidades de seguridad nacional o de orden público invocadas por Israel, aplicables en su totalidad o en parte a la construcción del muro. La Corte declara no estar convencida de estos fundamentos dados por Israel, pero no examina ningún elemento de hecho o de prueba que pudiere refutar en forma específica los argumentos fundados en los imperativos militares o las necesidades de seguridad nacional alegadas por Israel. 


\section{Conclusión}

Luego de haber analizado y puesto de relieve los principales puntos de conflicto que rodean la disputa en torno a la ciudad de Jerusalén, es posible afirmar que el objetivo de ambas partes, Israel, por un lado y la Autoridad Palestina, por otro, consiste en ejercer soberanía sobre el territorio de la ciudad. El punto principal del conflicto es Jerusalén Este. Las implicancias históricas y religiosas que tiene este sector de la ciudad, parecen constituir un punto neurálgico, respecto del cual ninguna de las partes está dispuesta a ceder. Palestina no se muestra conforme con la posición israelí que considera que debe constituir como capital del futuro Estado palestino a la ciudad de Ramallah u otra ciudad situada en territorio palestino, pero no en Jerusalén. Por su parte, los representantes palestinos insisten en que su futura capital debe estar localizada, aunque sea en una porción de la ciudad de Jerusalén, en especial dentro de la parte Este. Esta última opción constituye un punto neurálgico del conjunto de reclamos palestinos ante el Estado de Israel y la comunidad internacional. El Estado israelí ha adoptado una posición intransigente en este sentido, no permitiendo que la contingente capitalidad palestina sobre territorio jerosolimitano constituya un punto de futuras negociaciones, tanto a nivel multilateral como bilateral.

El rol de la comunidad internacional en este conflicto resulta de suma importancia, por lo tanto, es necesario que aquellos países con mayor influencia sobre las partes involucradas en el conflicto insten a las mismas a lograr una solución pacífica del diferendo. En relación con los métodos de solución de conflictos entre Estados, las partes pueden optar por cualquiera de ellos. Cuentan con sendos métodos de resolución de conflictos (Barboza, 2001) para dar un fin a la controversia. Los métodos (Brownlie, 2009) de tipo adjudicativo dentro de los cuales se incluyen el arbitraje y la solución judicial, podrían llegar a presentar algún obstáculo, ya que implicaría como punto final de la controversia una decisión única y final sobre el asunto proveniente de un tercero imparcial, ajeno a las partes, el cual impone una decisión que debe ser acatada. Esto provocaría en la concepción de las partes en conflicto una idea de falta de disposición y libertad durante el proceso para poder acordar ciertos puntos. Los métodos (Vinuesa, 1983 de tipo no adjudicativo, como las negociaciones, los buenos oficios o la conciliación, entre otros, podrían tener más éxito si se tiene en cuenta que implican una libertad para las partes en relación con el procedimiento a seguir, a los puntos a debatir, plazos, esquema de encuentros y una decisión final consensuada hasta el último momento de las negociaciones. Sin embargo, el primer obstáculo que se halla es la falta de respuesta tanto de una como de otra parte frente a las diferentes propuestas que se han intentado llegar a lo largo de la historia.

Las partes deben ser conscientes de que el hecho de sentarse a negociar implica ceder, hacer concesiones, pero que éstas son para el beneficio de ambos. De esta manera 
podrán llegar a una solución, respetando sus derechos logrando satisfacer los intereses de sus nacionales. Como se ha podido observar, se han ideado varias opciones para lograr que las dos partes en conflicto estén conformes con sus reclamos. A pesar de ello, siempre se han rechazado o no han alcanzado a materializarse por diversos motivos. En muchos casos fue por la intransigencia de ciertos gobernantes o por la falta de diálogo entre las partes, pero lo cierto es que ya se han dejado agotar varias oportunidades en donde seguramente con un poco de esfuerzo se hubiera podido llegar a un acuerdo beneficioso para las dos partes.

Para concluir, no hay que dejar de lado, el hecho de que Israel es una potencia ocupante (Haupais, 2007) de acuerdo con las normas de Derecho Internacional Humanitario (Gutiérrez Posse, 2014), por lo tanto, hay ciertos deberes (Ribeiro Roriz, Fernandes Carvalho Veçoso, Da Silva Tasquetto, 2013) que tiene que cumplir con la población palestina en los territorios ocupados, incluyendo Jerusalén Este. En este sentido, el respecto y la protección de los derechos humanos de los individuos que allí residen deben ser garantizadas (Ratner, 2005). La construcción del muro (Kimhi , 2006) ha provocado serios inconvenientes que deberán ser revisados por parte del gobierno israelí de manera tal que los palestinos que residen en las zonas que fueron afectadas por la división provocada por el muro puedan gozar del derecho a la libre circulación, al suministro de agua y a la libertad de trabajo, tal como ha sido señalado por la Corte Internacional de Justicia, en la mencionada Opinión consultiva sobre la construcción del muro en territorio palestino ocupado. Más allá de las noticas que aparecen diariamente en los medios de comunicación acerca de la rivalidad entre palestinos e israelíes lo cierto es que ambas comunidades conviven hace tiempo en Jerusalén y que esta convivencia demuestra que pueden llegar a una solución conjunta ya que las diferencias que en apariencia parecieran tener no son tantas, sino que por el contrario son más las similitudes, las que unen a estos dos pueblos que comparten dos religiones distintas pero unidas por una misma historia.

\section{Referencias Biblográficas}

Barboza, J. (2001). Derecho Internacional Público. Buenos Aires: Zavalia, $2^{\circ}$ edición.

Berkovitz, S. (2001). The Temple Mount and the Western Wall in Israeli Law. Jerusalén: The Jerusalem Institute for Israel Studies.

Brownlie, I. (2009).The Peaceful Settlement of International Disputes. Chinese Journal of International Law, 8(2), 267-283.

Esposito, C. (1996). La jurisdicción consultiva de la Corte Internacional de Justicia. Madrid: McGraw-Hill. 
Gold, D. (2007). The Fight for Jerusalem. Radical Islam, the West, and the Future of the Holy City. Washington: Regnery Publishing, Inc.

Gutierrez posse, H.D. (2014). Elementos de Derecho Internacional Humanitario. Buenos Aires: Eudeba, $1^{\circ}$ edición.

Haupais, N. (2007). Les obligations de la puissance occupante au regard de la jurisprudence et de la pratique récentes. Revue Générale de Droit International Public, 111(1), 117-147.

Kimhi, I. (2006). The operational regime of the security fence around Jerusalem: Potential implications for the city. En Kimhi, I. (Ed), The Security Fence around Jerusalem: Implications for the City and Its Residents (pp.15-31). Jerusalén: Jerusalem Institute for Israel Studies.

Kimhi, I., Choshen, M., y Assap-shapira, Y. (2006). Jerusalem as a Component of Israel's National Strength, Indicators of the State of the Capital, and a View to the Future. Jerusalén: Jerusalem Institute for Israel Studies.

Klein, M. (2001). Jerusalem the contested city. Jerusalén: Jerusalem Institute for Israel Studies.

Kuete Minga, R. (1997). Le Traité de paix du 26 octobre 1994 entre l'État d'Israël et le Royaume Hachémite de Jordanie. Revue Générale de Droit International Public, 101(2), 391-421.

Lehrs, L. (2011). Peace talks over Jerusalem. Review of the Israeli-Palestinian Negotiations Concerning Jerusalem 1993-2011. Jerusalén: Jerusalem Institute for Israel Studies.

Mouton, J.D. (1996). L'autonomie palestinienne après l'accord intérimaire israélo-palestinien du 28 septembre 1995. Revue Générale de Droit International Public, 100(4), 951-977.

Poissonnier, G., y Osseland, P. (2010). Les colonies israéliennes de Cisjordanie en droit international. Revue Générale de Droit International Public, 114(1), 63-92.

Ratner, S.R. (2005). Foreign Occupation and International Territorial Administration: The Challenges of Convergence. The European Journal of International Law, 16(4), 695-719.

Ribeiro Roriz, J.H., Fernandes Carvalho Veçoso, F., y Da Silva Tasquetto, L. (2013). A administração de territórios ocupados: indeterminação das normas de direito internacional humanitário? Revista de direito internacional, 10(2), 183-195. 
Shragai, N. (2010). Jerusalem Master Plan: Demography, Geopolitics, and the Future of Israel's Proposed Master Plan. Jerusalén: Jerusalem Center for Public Affairs.

Shragai, N. (2012). Jerusalem: Correcting the International Discourse. How the West gets Jerusalem wrong. Jerusalén: Jerusalem Center for Public Affairs.

Vinuesa, R. (1983). La solución pacífica de controversias entre Estados. Lecciones y Ensayos, (4), 7-32.

Virally, M. (1963). Droit international et décolonisation devant les Nations Unies. Annuaire français de droit international, (9), 508-541. 